\title{
THE EFFECT OF OSMOTIC AND ION-OSMOTIC STRESSES ON THE BLOOD AND URINE COMPOSITION AND URINE FLOW OF RAINBOW TROUT (SALMO GAIRDNERI)
}

\author{
PAUL T. KosteCKI* \\ School of Natural Resource, University of Michigan, Ann Arbor, MI 48109, USA
}

(Received 8 November 1983)

\begin{abstract}
Changes in urine and plasma concentrations (sodium, potassium, magnesium, calcium and total osmotic) and urine production were determined in fish exposed to various concentrations of an ionically active substance, sodium chloride, and a non-electrolyte, mannitol, as well as freshwater.

2. Responses occurred for the most part over a short crisis period preceeding establishment of new stable conditions.

3. It was shown that plasma homeostasis was not maintained in response to changing ion-osmotic and osmotic gradients.

4. Urinary osmotic and ionic concentrations were unaffected and urine production was shown to be inversely related to the external concentration.

5. It is suggested that ionic shifts between body compartments are an important aspect of ion-osmotic adaptation.
\end{abstract}

\section{INTRODUCTION}

Several studies have been made on ionic and osmotic regulation in fish (Gordon, 1963; Weisbart, 1967; Wood and Randall, 1973a, b; Beyenbach and Kirschner, 1975; Talbot et al., 1982; Holeton et al., 1983; King and Goldstein, 1983). Most of this work has been concerned with regulation in fish fully adjusted to freshwater or seawater and then exposed to a salinity challenge. Few observations have been made on regulatory changes during the transition period for fish rapidly transferred to different ionosmotic concentrations (Bath and Eddy, 1979; Kostecki, 1979, 1982).

The purpose of this paper is to determine the ionic and osmotic responses during ion-osmotic regulation in fish exposed to various ion-osmotic (sodium chloride) and osmotic (mannitol) concentrations during the initial exposure and after 7 days of acclimation.

\section{MATERIALS AND METHODS}

Hatchery rainbow trout (Salmo gairdneri), weighing $100-250 \mathrm{~g}$, were held in dechlorinated freshwater for at least 4 weeks prior to experimentation. Fish were fed daily, to satiation, with Purina trout chow and acclimated and tested at a water temperature of $15.0 \pm 0.1^{\circ} \mathrm{C}$. The fish were starved for 7 days prior to any given experiment.

Experiments were performed to measure the responses of plasma and urine osmolality and ionic composition to changes in external osmotic (mannitol) and ion-osmotic (sodium chloride) concentrations at ambient concentrations above (250 and 500 milliosmoles (mOsm)) and below (deionized and freshwater and $10 \mathrm{mOsm}$ ) the plasma isosmotic level. Observations on blood covered a short-term $(36 \mathrm{hr})$ and long-term ( 7 days) period, and on urine, a 4 day period.

Two types of experiments were performed to minimize any stress effects due to sampling. One group of fish was used to measure ion-osmoregulation changes in the blood (blood group) under various ion-osmotic concentrations,

*Present Address: Zoology Department, University of Massachusetts, Amherst, MA 01003, U.S.A. while a second group was used to measure changes in the urine (urine group). The blood group was further divided into a short-term blood group and a long-term blood group. The long-term blood group and urine group were used to measure changes over 7 and 4 days of exposure, respectively. The short-term blood groups allowed for more precise sampling during the first $36 \mathrm{hr}$ when most blood changes probably occurred in fish exposed to sodium chloride (Kostecki, 1978).

The experimental solutions, sodium chloride or mannitol, were made by adding the appropriate chemical to deionized water to yield the necessary concentrations. Samples of the deionized water as well as the experimental solutions were analyzed to insure proper osmotic and ionic (sodium, potassium, calcium and magnesium) concentrations. In the experimental solution, the calcium concentration was $1.5 \mathrm{mEq} \mathrm{l}^{-1}$ and the potassium and magnesium concentrations were essentially zero. The sodium concentration was zero in the mannitol media, but, of course, varied in the sodium chloride solutions. The calcium, magnesium and potassium gradients between the fish and the external environment remained constant despite the changes in osmolality.

\section{Blood}

Fish, weighing $100-150 \mathrm{~g}$, for the long-term experiment were transferred to $22,16-1$ polypropylene tanks at a density of seven fish/tank. The fish were allowed to acclimate to the test tanks in aerated freshwater for $24 \mathrm{hr}$. The experiments were started after this acclimation period with an initial sample (Day 0) just prior to changing the external concentration. The tanks were then divided into seven groups of: distilled water and three concentrations each of sodium chloride and mannitol of 10,250 and $500 \mathrm{mOsm}$.

Usually, five fish, with two exceptions, were sampled from each concentration after $1,2,4$, and 7 days of exposure. Mortality dictated that only four fish were sampled from the 250 mOsm sodium chloride group on the seventh day, and three and two fish from the 500 mOsm sodium chloride group on days 4 and 7 , respectively.

Fish for the short-term experiment were transferred to 20 similar tanks at a density of five to six fish/tank. They were allowed to acclimate to the tanks for $24 \mathrm{hr}$ in freshwater. After the acclimation period an initial sample was taken and the tanks divided into two groups; freshwater and 


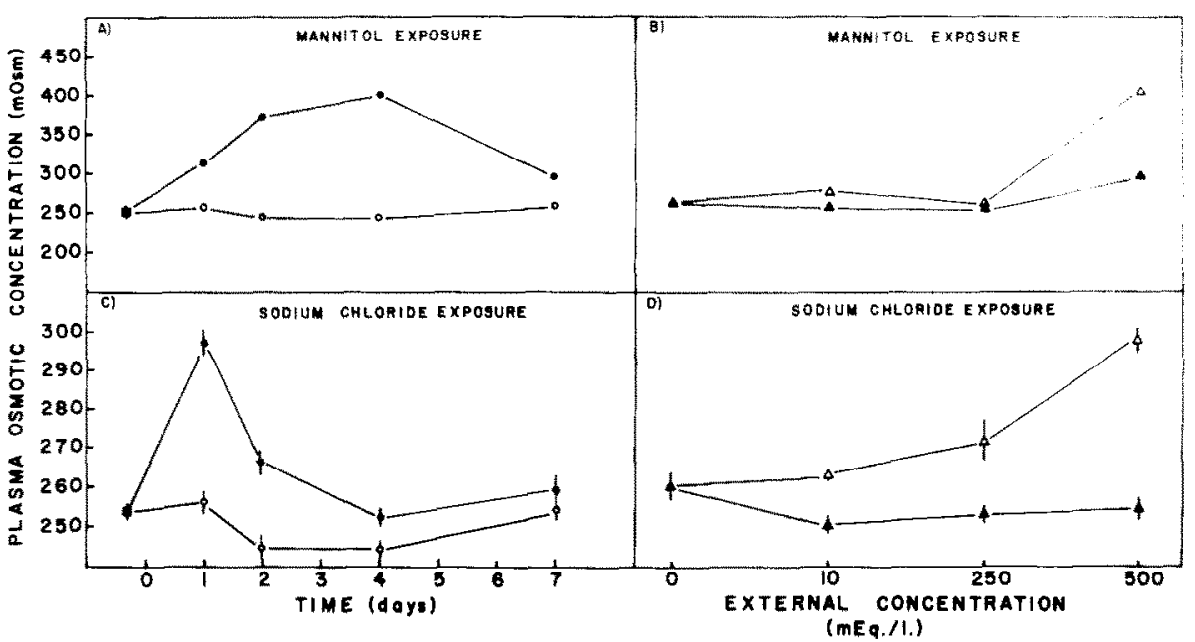

Fig. 1. Typical responses of rainbow trout exposed to mannitol and sodium chloride. (A) Exemplary relationship between plasma osmotic concentration and time for fish exposed to ambient concentrations of $500 \mathrm{mOsm}$ mannitol (shaded symbols) and deionized water (unshaded symbols). (B) Relationship between maximum plasma osmotic concentration (unshaded symbols) during exposure of fish to various ambient mannitol concentrations and levels after 7 days (shaded symbols). (C) Exemplary relationship between plasma osmotic concentrations and time for fish exposed to ambient concentrations of $500 \mathrm{mOsm}$ sodium chloride (shaded symbols) and deionized water (unshaded symbols). (D) Relationship between maximum plasma osmotic concentration (unshaded symbols) during cxposure of fish to various ambicnt sodium chloride concentrations and level after 7 days (shaded symbols). Bars \pm 2 S. E., $n=5$ except (C) $n=3$ for Day 4 and $n=2$ for Day 7 for 500 mOsm sodium chloride. (D) $n=4$ for 250 mOsm and $n=2$ for $500 \mathrm{mOsm}$.

$200 \mathrm{mOsm}$ sodium chloride. Five fish were sampled from each concentration every $4 \mathrm{hr}$ for $36 \mathrm{hr}$.

The medium in each tank for both sets of experiments was changed every $24 \mathrm{hr}$ to prevent the buildup of waste products. Blood samples were obtained by cardiac puncture from anesthesized (MS 222; 1:20.000) fish. Ammonium heparin was used to prevent clotting and hemolysis. The blood samples were centrifuged at $5000 \mathrm{rev} / \mathrm{min}$ for $10 \mathrm{~min} \pm 1 \mathrm{~min}$. The plasma portion was stored in acidwashed, polypropylene vials at $-20 \mathrm{C}$ until analysis. Hematocrit was also measured.

\section{Urine}

Urinary catheters were implanted in fish. $150-250 \mathrm{~g}$, using the technique described by Kostecki (1978). The technique was improved by the construction of catheters from Silastic medical grade tubing $(0.030 \times 0.650 \mathrm{in})$. After implanting the catheters the fish were transferred to respirometers (Kostecki, 1979) allowing urine collection in $50 \mathrm{mll}$ volumetric flasks. Urine was collected for fish exposed to: freshwater, deionized water and three concentrations each of sodium chloride and mannitol of 10.250 and $500 \mathrm{mOsm}$. Urine samples were collected from five fish from freshwater and four fish from each of the other concentrations except for three fish from the $500 \mathrm{mOsm}$ sodium chloride group because of mortality. The fish were exposed to freshwater for 4 days prior to exposure to the test concentrations, enabling recovery from the implantation (Kostecki, 1978). The 24-hr cumulative urine fow rate was measured laly for 4 days. The urine samples were stored in acid-washed polypropylene bottles at $-20 \mathrm{C}$ until analysis for urine osmotic concentration and ionic composition

\section{Analytical procedures}

Osmotic concentrations of urine and plasma samples were determined against appropriate dilutions of prepared standards using a Model 512A. Wescor vapor pressure osmometer. Ionic analysis of $\mathrm{Na}^{*}, \mathrm{~K}^{-}$and $\mathrm{Mg}^{2-}$ determinations, both standards and unknowns were diluted with lanthanum solution $\left(0.1^{\circ} / \mathrm{w} / \mathrm{v} \mathrm{La}\right)$ to atvoid $\mathrm{Ca}^{2+}$ interference. The significance of the differences among means of samples was tested using a multiple range test, Student-Newman-Kuel, at the $P<0.05$ level.

\section{RESUITS}

Plasma osmotic concentration of fish exposed to sodium chloride increased proportionally to the external concentration over the first $1-2$ days, as illustrated for the 500 mOsm sodium chloride group in Fig. 1(C, D) but returned to pre-exposure levels after 7 days. These plasma osmotic increases were primarily due to increases in sodium and potassium concentrations [Figs 2(C), 3, 4]. Plasma calcium and magnesium concentrations decreased in fish exposed to the hypo- and hyper-ion-osmotic sodium chloride concentrations (Fig. 4).

Plasma osmotic concentrations were unaffected by external hypo-osmotic $(0$ and $10 \mathrm{mOsm})$ and slightly hyper-osmotic ( $250 \mathrm{mOsm}$ ) concentrations of mannitol and increased significantly $(P<0.05)$ at the hyper-osmotic concentration $(500 \mathrm{mOsm})$ [Figs. I(A. $B)$ ] but without a parallel increase in any of the plasma ions measured [Figs 2(A, B)]. Plasma sodium. potassium and magnesium in lish exposed to various mannitol concentrations remained fairly constant, but plasma calcium decreased significantly $(p<0.05)$ in response to the various mannitol concentrations [Figs 2(B), 5 and 6].

The initial changes in plasma osmotic and ionic concentrations and hematocrit were evaluated in greater detail over the short-term $(0-36 \mathrm{hr})$ for fish exposed to an elevated sodium chloride concentration ( $200 \mathrm{mOsm})$. Plasma osmotic concentration of fish exposed to sodium chloride increased to levels significantly higher $(P<0.05)$ than fish exposed to freshwater after $4 \mathrm{hr}$ [Fig. 7(A)]. Thereafter, plasma osmotic concentration decreased to levels only 


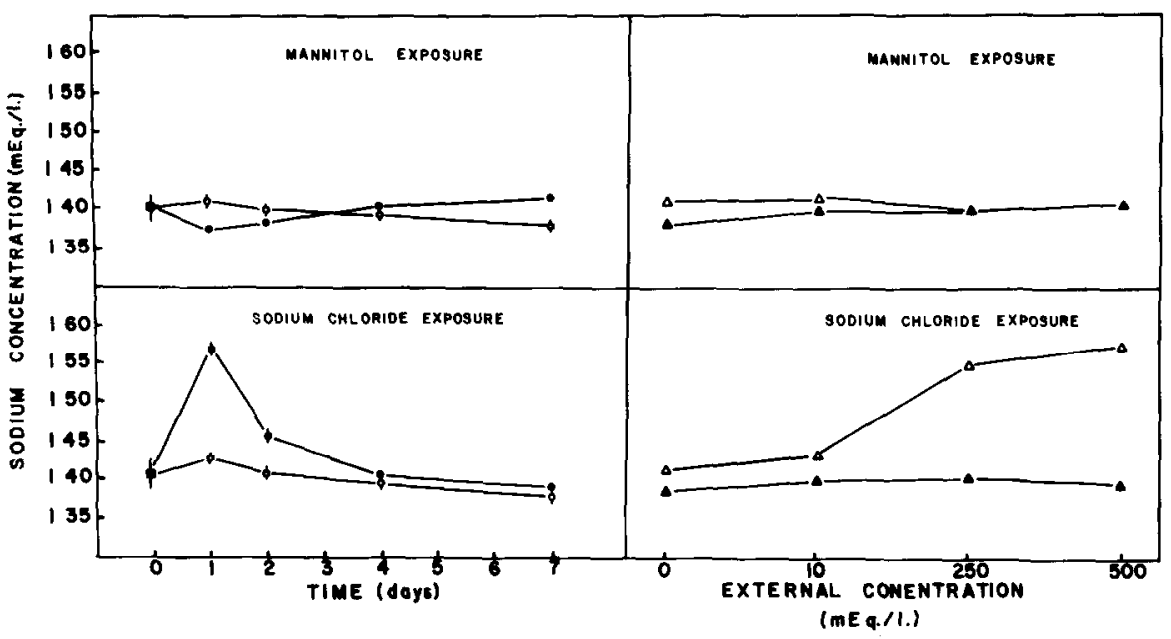

Fig. 2. Typical responses of rainbow trout exposed to mannitol and sodium chloride. (A) Exemplary relationship between plasma sodium concentration and time for fish exposed to ambient concentrations of $500 \mathrm{mOsm}$ mannitol (shaded symbols) and deionized water (unshaded symbols). (B) Relationship between maximum plasma sodium concentration (unshaded symbols) during exposure of fish to various ambient mannitol concentrations and levels after 7 days (shaded symbols). (C) Exemplary relationship between plasma sodium concentration and time for fish exposed to ambient concentrations of $500 \mathrm{mOsm}$ sodium chloride (shaded symbols) and deionized water (unshaded symbols). (D) Relationship between maximum plasma sodium concentration (unshaded symbols) during exposure of fish to various ambient sodium chloride concentrations and levels after 7 days (shaded symbols). Bars \pm S.E., $n=5$ except (C) $n=3$ for Day 4 and $n=2$ for Day 7 for 500 mOsm sodium chloride, (D) $n=4$ for 250 mOsm and $n=2$ for 500 mOsm.

slightly higher than the control and pre-exposure levels by $36 \mathrm{hr}$. The higher plasma osmotic concentration of fish exposed to the sodium chloride appears to be due, in part, to an increase in plasma sodium [Fig. 7(B)] and, to a lesser extent, plasma potassium [Fig. 7(C)]. Plasma calcium [Fig. 7(D)] and magnesium [Fig. $7(\mathrm{E})]$ concentrations in fish exposed to

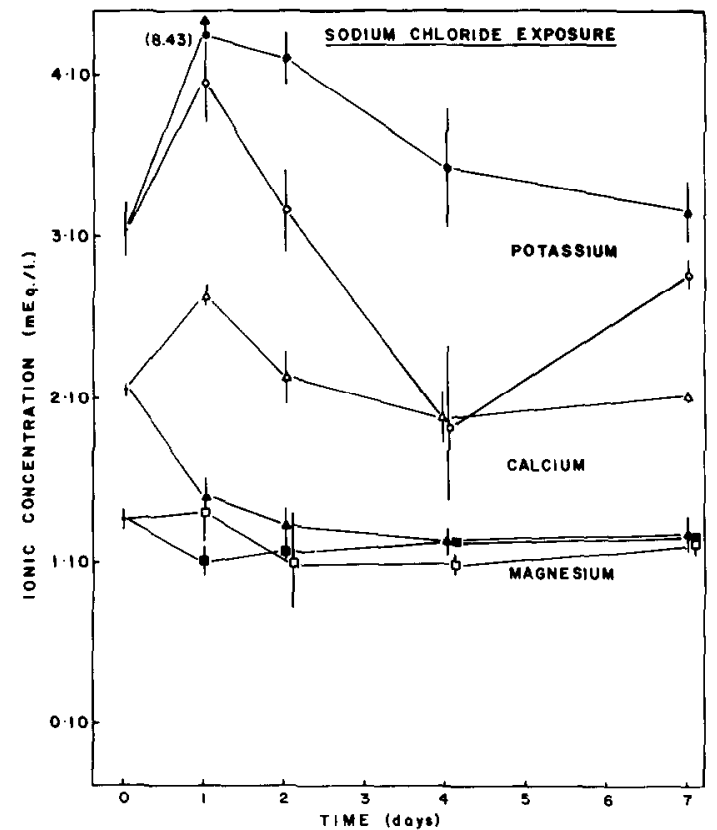

Fig. 3. Exemplary relationship between plasma potassium, calcium and magnesium concentrations and time for rainbow trout exposed to ambient concentrations of $500 \mathrm{mOsm}$ sodium chloride (shaded symbols) and deionized water (unshaded symbols). Bars +2 S.E., $n=5$ except $n=3$ for Day 4 and $n=2$ for Day 7 for 500 mOsm sodium chloride. sodium chloride decreased and remained significantly lower $(P<0.05)$ than the controls after $12-16 \mathrm{hr}$ of exposure. No difference in calcium and magnesium gradients existed between the freshwater and the sodium chloride medium that could explain this change. Since absolute concentration changes were small, these ions may be qualitatively involved in

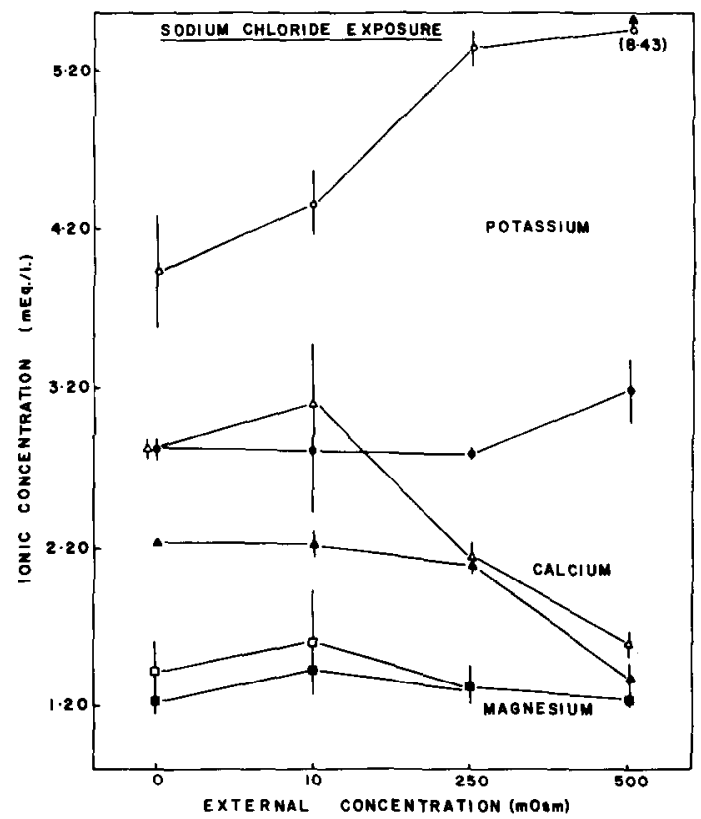

Fig. 4. Relationship between maximum plasma potassium, calcium and magnesium concentrations (unshaded symbols) during exposure of rainbow trout to various sodium chloride concentrations and levels after 7 days (shaded symbols). Bars \pm 2 S.E., $n=5$ except $n=4$ for 250 mOsm and $n=2$ for $500 \mathrm{mOsm}$. 


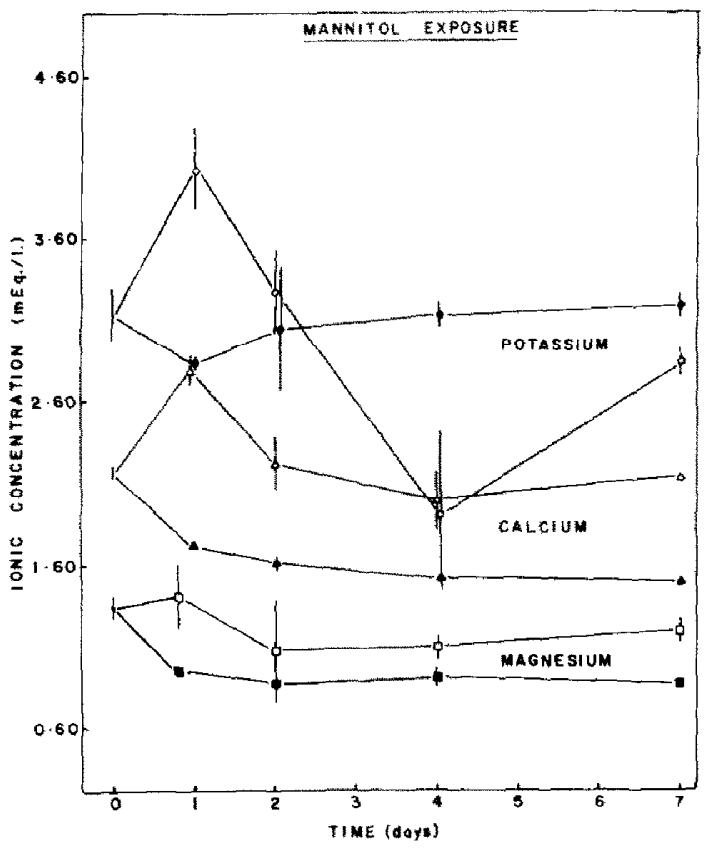

Fig. 5. Exemplary relationship between plasma potassium. calcium and magnesium concentrations and time for rainbow trout exposed to ambient concentrations of $500 \mathrm{mOsm}$ mannitol (shaded symbols) and to delonized water (unshaded symbols). Bars \pm 2 S.E. $n=5$.

osmotic or ion-osmotic regulation, perhaps by affecting membrane permeability.

Fish exposed to sodium chloride had significantly lower $(P<0.05)$ hematocrits after $8 \mathrm{hr}$ of exposure [Fig. 7(F)] than the control fish for the duration of the experiment.

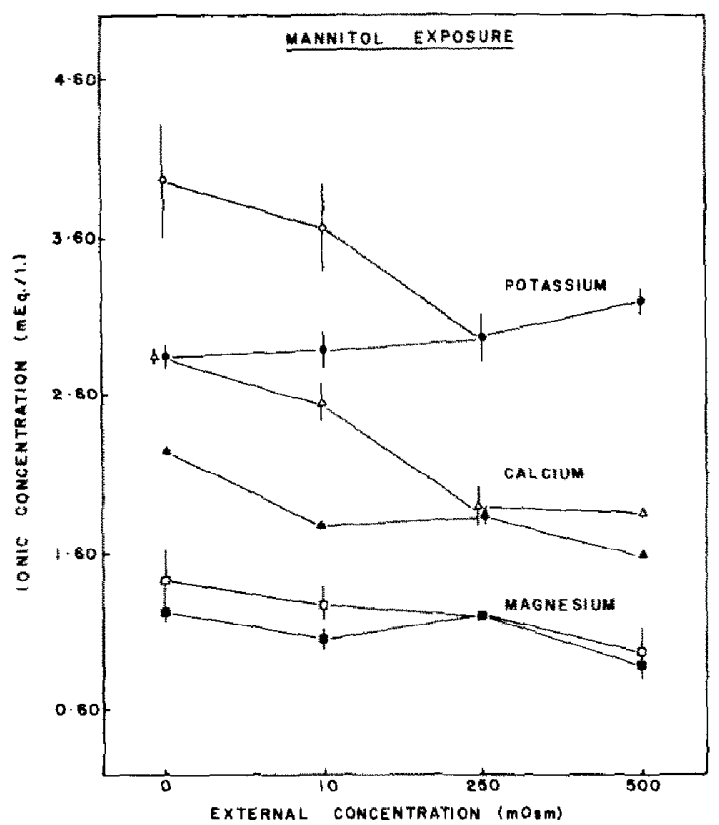

Fig. 6. Relationship between maximum plasma potassium, calcium and magnesium concentrations (unshaded symbols) during exposure of rainbow trout to various mannitol concentrations and level after 7 days (shaded symbols). Bars +2 S.E., $n=5$

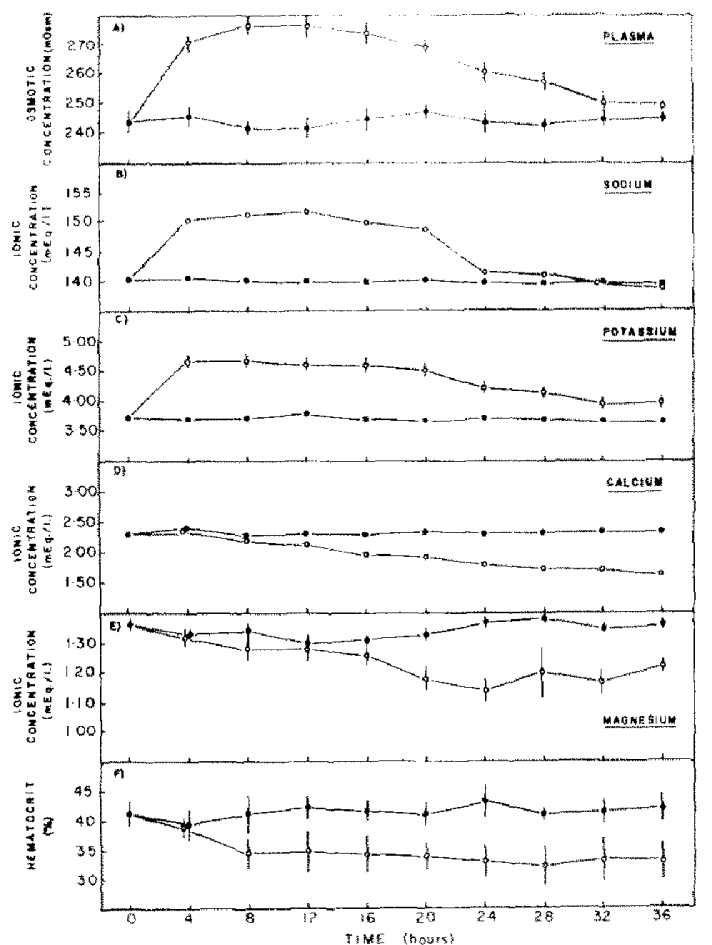

Fig. 7. Responses of rainbow tront exposed to treshwater and sodium chloride. (A) Relationship between plasma osmotic concentration and time for fish exposed for $36 \mathrm{hr}$ to freshwater (shaded symbols) and $200 \mathrm{mOsm}$ sodium chloride (unshaded symbols). (B) Relationship between plasma sodium concentration and time for fish exposed for $36 \mathrm{hr}$ to freshwater (shaded symbols) and $200 \mathrm{mOsm}$ sodium chloride (unshaded symbols). (C) Relationship between plasma potassium concentration and time for fish exposed for $36 \mathrm{hr}$ to freshwater (shaded symbols) and $200 \mathrm{mOsm}$ sodium chloride (unshaded symbols). (D) Relationship between plasma calcium concentration and time for fish exposed for 36 hr to freshwater (shaded symbols) and $200 \mathrm{mOsm}$ sodium chloride (unshaded symbols). (E) Relationship between plasma magnesium concentration and time for fish exposed for $36 \mathrm{hr}$ to freshwater (shaded symbols) and $200 \mathrm{mOsm}$ sodium chloride (unshaded symbels) (F) Relationship between hematocrit and time for fish exposed for $36 \mathrm{hr}$ to freshwater (shaded symbols) and $200 \mathrm{mOsm}$ sodium chloride (unshaded symbols). Bars \pm S.E., $n=5$.

The urine flow rate and composition also varied with exposure time and concentration. Urine flow decreased after implantation of catheters and reached stable rates as measured in the control (fresh water) group by the fourth day [Fig. 8(A)]. This is slightly longer than the 3 day period previously reported by Kostecki (1979). These data suggest that the fish recovered from stress due to catheter implantation by Day 4 (McKim, 1968).

The rate of urine production decreased over time at the higher external concentrations [Fig. 8(A, C)]. However, the reduction was less in fish exposed to mannitol [Fig. 8(B)] than it was in those tested in sodium chloride [Fig. 8(D)] solutions.

Osmotic concentration of the urine showed very little change in fish exposed to any external concentration, either with mannitol or sodium chloride. The urine osmotic concentration did not exceed 3 mOsm (the minimal resolution level of the analyses) in any 


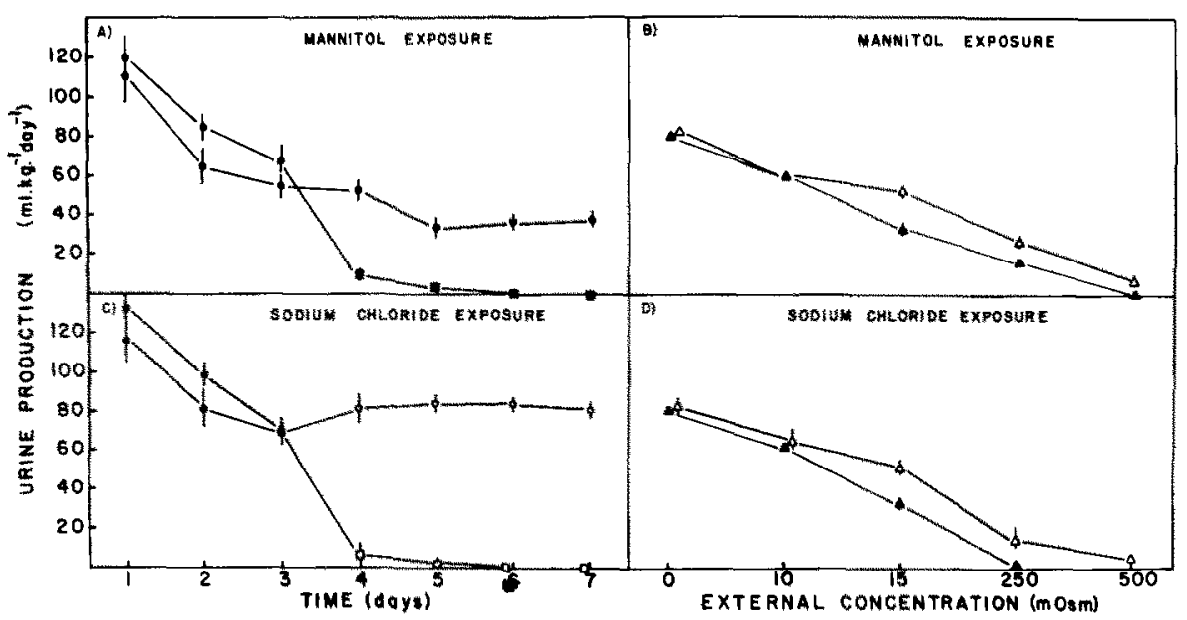

Fig. 8. Typical responses of rainbow trout exposed to mannitol and sodium chloride. (A) Exemplary relationship between urine production and time for fish exposed to ambient concentrations of $500 \mathrm{mOsm}$ mannitol (shaded squares) and freshwater (shaded circles). (B) Relationship between maximum urine production (unshaded symbols) during exposure of fish to various ambient mannitol concentrations and levels after 4 days (shaded symbols). (C) Exemplary relationkhip between urine produetion and time for fish exposed to ambient concentrations of $500 \mathrm{mOsm}$ sodium chloride (unshaded squares), deionized water (unshaded circles) and freshwater (shaded circles). (D) Relationship between maximum urine production (unshaded symbols) during exposure of fish to various ambient sodium chloride concentrations and levels after 4 days (shaded symbols) Bars $2 \pm$ S.E., $n=5$ except (C) $n=3$ for 500 mOsm sodium chloride and (D) $n=3$ for 500 mOsm sodium chloride.

group of fish. This finding is contrary to other reports by investigators, who have shown that urine concentration of some fish varies drastically in response to changes in the external salinity (Lahlou, 1967). Only exemplary data of the major trends are presented for the purpose of this paper. The reader is directed to Kostecki (1981) for the complete data set.

\section{DISCUSSION}

The fish were experimentally exposed to two sets of situations; changes in the osmotic gradient with no change in the ionic gradient (mannitol) and simulta* neous changes in both the osmotic and ionic gradients (sodium chloride and deionized water). The only ionic gradient that was manipulated was the sodium (and chloride) gradients; potassium, calcium and magnesium being held constant in the deionized water, sodium chloride and mannitol media.

However, despite only two ambient variables being altered, fish responses varied over time and according to the ambient concentration above and below plasma osmotic concentration. These time dependent responses occurred over a short crisis period preceding establishment of new stable conditions. Concentration-related responses depended on whether the medium was more or less concentrated than the plasma. For example, a hyper-osmotic external mannitol solution induced an increased plasma osmotic concentration, whereas an equal hypogradient had no such cffcet. Similarly, greater effects on plasma ions occurred with hyper-ion-osmotic solutions than with hypo-solutions. Therefore, each of these responses, as thcy relatc to hypo- and hyper-conditions are discussed separately.

\section{Short-term experiments-crisis period}

The observations on fish exposed to changes in ionic and osmotic gradients (sodium chloride) ex- hibited a "crisis" period (Bath and Eddy, 1979) when large changes occurred in the plasma (24 $48 \mathrm{hr}$ after exposure). This period was therefore examined in detail by the shortuterm experiments. As expected maximum values for plasma osmotic, potassium and sodium concentrations and hematocrit occurred during the crisis period [Fig. 7(A-C, F)]. The crisis period was followed by a stabilization period, when slower physiological changes occurred. The stabilization period appeared to be of a shorter duration than previously reported for smaller trout (Bath and Eddy, 1979). Stabilization appeared to be almost complete with respect to plasma osmotic, sodium and potassium concentrations after $36 \mathrm{hr}$ of exposure. However, no crisis period and, hence, no stabilization period for plasma calcium and magnesium concentrations occurred [Fig. 7(D, E)].

A fish in freshwater is confronted by problems of water influx and salt loss because of the nature of the osmotic and ionic gradients. The addition of sodium chloride up to $200 \mathrm{mOsm}$ was below the isosmotic level of the fish $(244 \pm 5 \mathrm{mOsm})$. At this concentration there was $\left.99 \mathrm{mEq}\right|^{-1}$ sodium in solution as compared to $141 \mathrm{mEq}^{-1}$ sodium in the fish plasma. Thus, the external concentration only reduced the osmotic and sodium gradients, but did not reverse them with respect to the control fish. This change would be expected to reduce regulatory problems, but, in practice, the increase in plasma osmotic and sodium concentrations indicate greater imbalance in plasma levels. Therefore, ionic and osmotic regulatory mechanismus appear to lag in response to the changing external concentrations.

Urine flow is the primary source of water regulation in freshwater fish and it quickly decreased in fish exposed to clevated sodium chloride levels [Fig. 8(D)]. Ionic losses are minimized by active reabsorption in the kidney and losses are replaced by 
active uptake at the gills. The plasma sodium concentration and increases in plasma osmotic concentration would be explained if there was a lag time in compensation for the new gradient. The active transport mechanisms would proceed at the same rate and in the same direction as in freshwater after the external sodium concentration was increased. This lag time would be the "crisis" period

The gradual decrease in plasma calcium and magnesium concentrations may be due to their role in passive, long-term ion-osmoregulation through their involvement in permeability changes. Lowenstein et al. (1967) have shown that the permeability of membrane surfaces in Chironomus salivary glands depends on the calcium and magnesium ion concentrations. It has been known for some time that calcium ions may contribute to a decreased permeability and reduce water and ion fluxes in some fish (Kirschner, 1969: Motais et al., 1965; Potts and Fleming, 1970). Smith (1970) showed that Nereis diversicolor was unable to osmoregulate in calcium-free brackish water. Eels are unable to osmoregulate in seawater and die of dehydration in calcium-free seawater (Sharatt et al. 1964).

The exact nature of the effect of calcium on permeability is not known. It has been suggested that calcium reduces the hydration of polar organic molecules by reducing the repulsion between fixed anions. This results in a closer packing of the organic molecules and, hence, a reduction in permeability. It has also been suggested that the permeability of the body surface depends not only on the permeability of the constituent cells but also on the permeability of the intercellular cement (Shanes, 1960; Houston, 1964; Lowenstein et al., 1967). Calcium stabilizes the intercellular cement making it less permeable.

The decrease in hematocrit in trout exposed to sodium chloride probably occurred as a result of the increase in plasma osmotic concentration. This increase would cause water to be osmotically withdrawn from the erythrocytes, reducing their size. Thus, the size reduction of individual erythrocytes would decrease the hematocrit.

\section{Long-term hyper-osmotic and hyper-ion-osmotic ex- periments}

Fish exposed to hyper-osmotic (mannitol) and hyper-ion-osmotic (sodium chloride) solutions showed increases in plasma osmotic concentration [Fig. 1(B, D)]. The osmotic gradient for fish exposed to these concentrations is such that the increase in plasma osmotic concentration is likely due to an osmotic dehydration of the plasma. However, osmotic dehydration would cause hemoconcentration; i.e. an increase in plasma ionic concentration. The absence of an increase in plasma ions for fish exposed to hyper-osmotic solutions must therefore be associated with transport of these ions either into storage compartments or out of the animal. This transport would require energy which could account for the significant increase in oxygen consumption in fish exposed to hyper-osmotic mannitol solutions (Kos* tecki, 1981). The constant ion concentration, but increased osmotic concentration suggests that the increase in plasma osmotic concentration was likely due to large non-diffusible molecules.
In contrast, the increase in plasma osmotic concentration for fish exposed to hyper-ion osmotic solutions can be largely attributed to increased plasma ions. If a concomitant increase in anions is assumed to maintain electrical parity, sodium, potassium. calcium and magnesium increases account for $90^{2 \prime}{ }^{2}$ of the plasma osmotic concentration increase in fish exposed to $500 \mathrm{mOsm}$ sodium chloride. Thus, of the $44 \mathrm{mOsm}$ increase in plasma osmotic concentration about $40 \mathrm{mOsm}$ was due to ions and presumably 4 mOsm to non-diffusible molecules in these fish. This, of course, is an oversimplification since the ionic concentrations contributing to the osmotic concentration vary due to a number of factors, for example. Gibbs-Donnan equilibria and, ultimately, their activity. Thus the calculations serve only as qualitative estimates of the osmotic contribution of large non-diffusible molecules.

The increase in plasma osmotic concentration in fish exposed to the hyper-ion-osmotic solutions is presumably due to the plasma sodium concentration exceeding the excretion rates plus some hemoconcentration as in the hyper-osmotic mannitol concentration.

Ion transport in the adaptation of fish to high salinities has been shown not only to include extrarenal monovalent ion excretion and renal bivalent ion excretion, but also shifts in tissue and extracellular fluid ionic content (Miles et al., 1968; Bath and Eddy. 1979). The shift of ions to body compartments (e.g. interstitial fluid) other than the plasma has been largely ignored in the analyses of energy expenditure for ionic regulation. However, the interstitial fluid comprises almost 7 times the volume of plasma in freshwater teleosts (Thorson, 1961). The relative magnitude of the interstitial volume could dampen plasma ionic concentration changes but substantially add to energy expenditure during adaptation of fish to high salinities. The processes of excretion and shifting both involve active transport.

Plasma sodium concentrations were not the only changes to occur under hyper-osmotic and hyper-ionosmotic conditions. Plasma calcium and magnesium concentrations of fish exposed to these conditions also declined (Figs. 4, 6), with no differences between mannitol and sodium chloride. The decrease in these ion levels was not due to excretion because urine ionic levels were unchanged and the rate of urine output declined. Therefore, the divalent ions must be regulated by tissue storage. The decrease in plasma calcium and magnesium concentrations may be due to their involvement with permeability changes. However, the similar decrease of these ions in response to osmotic as well as ion-osmotic changes suggests that if these ions are involved in permeability changes, it is with respect to water permeability rather than sodium and chloride ion permeability.

Plasma potassium concentrations remained relatively constant in fish exposed to various osmotic concentrations, suggesting that potassium is relatively inactive in osmotic regulation. However, plasma potassium of fish exposed to hyper-ionosmotic concentrations followed similar trends to sodium, increasing immediately after exposure but returning to pre-exposure levels by Day 7 (Fig. 3). Since the plasma potassium concentrations of fish 
exposed to ion-osmotic media paralleled plasma sodium, there may be some association of these two ions with the ion regulatory mechanisms.

Urine production decreased at hyper-osmotic and hyper-ion-osmotic concentrations and no increase occurred in urine concentration. Therefore, it appears that the kidney is not a major monovalent regulatory route at these concentrations. In contrast, some investigators have indicated that fish exposed to high salinities produce urine of high osmotic concentration (Lahlou, 1967). However, this was probably due to increased urine divalent ion concentration. These experiments were performed in seawater where all ions were increased simultaneously so that divalent influx would have been increased in addition to sodium chloride.

\section{Long-term hypo-osmotic and hypo-ion-osmotic ex- periments}

The hypo-osmotic and hypo-ion-osmotic situations present different problems and regulatory mechanisms. Excess water excretion together with bivalent ion regulation (conservation) occur in the kidney. Monovalent ion regulation (uptake) occurs at the gills. The large water gradient associated with both hypo-ion-osmotic concentrations (mannitol and sodium chloride) as well as freshwater concentrations is reflected in high production of copious urine. The urinary osmotic concentrations of fish exposed to these concentrations were below detectable levels, consistent with the low urinary ionic concentrations. Reabsorption through active transport would be required to maintain the low urinary ionic concentrations and, since urine is produced at a high rate, reabsorption could require substantial energy expenditure (Kostecki, 1981).

Thus it appears that ionic and osmotic regulation may involve ion excretion and transfers between body compartments. These transfers occur shortly $(12 \mathrm{hr})$ after exposure to different osmotic and ionosmotic concentrations and would be associated with energy expenditure for regulation. It also appears that calcium and, perhaps, magnesium may be involved in osmotic regulation and in terms of ionic regulation the kidney is of minor importance.

Acknowledgements-This study was supported by funds provided for graduate research in the University of Michigan School of Natural Resources and was submitted by Paul T. Kostecki in partial fulfillment of the Ph.D. requirements. The author would like to thank those who provided information and comments on the manuscript, Drs Paul W. Webb, William Dawson, Frank F. Hooper, Eugene Fritz and Ronald Drobney. In addition, Dr Paul W. Webb provided invaluable advice and assistance throughout all phases of the study.

\section{REFERENCES}

Bath R. N. and Eddy F. B. (1979) Salt and water balance in rainbow trout (Salmo gairdneri) rapidly transferred from fresh water to sea water. J. exp. Biol. 83, 193-202.

Beyenbach K. W. and Kirschner L. B. (1975) Kidney and urinary bladder functions of the rainbow trout in magnesium and sodium excretion. Am. J. Physiol. 229, 383-389.
Gordon M. S. (1963) Chloride changes in rainbow trout (Salmo gairdneri) adapted to different salinities. Biol. Bull. Mar. biol. Lab Woods Hole 124, 45-54.

Holeton G. F., Neumann P. and Heisler N. (1983) Branchial ion exchange and acid-base regulation after strenuous exercise in rainbow trout (Salmo gairdneri). Resp. Physiol. 51, 303-318.

Houston A. H. (1964) On passive features in the osmoregulatory adaptation of anadromous salmonids to sea water. J. exp. Biol. 44, 33-46.

King P. A. and Goldstein L. (1983) Organic osmolytes and cell volume regulation in fish. Molec. Physiol. 4, 53-66.

Kirschner L. B. (1969) Sodium fluxes in perfused eel gills. Am. J. Physiol. 217, 596-604.

Kostecki P. T. (1978) Energy expenditure for osmoregulation in rainbow trout, Salmo gairdneri. M.S. thesis, Univ. of Michigan, $55 \mathrm{pp}$.

Kostecki P. T. (1979) Energy expenditure for osmoregulation in rainbow trout, Salmo gairdneri. Mich. Acad. 12, $155-166$.

Kostecki P. T. (1982) Ionic and osmotic regulation in rainbow trout (Salmo gairdneri). Ph.D. dissertation, University of Michigan, $90 \mathrm{p}$.

Kostecki P. T. and Jones J. J. (1982) The effect of osmotic and ion-osmotic stresses on the mortality of rainbow trout (Salmo gairdneri). Comp. Biochem. Physiol. 74A, 773-775.

Lahlou R. (1967) Renal function in flounders. Comp. Biochem. Physiol. 30, 925-938.

Lowenstein W. R., Nakas M. and Socolar S. J. (1967) Junctional uncoupling: Permeability transformations at a cell membrane junction. J. gen. Physiol. 50, 1865-1892.

McKim J. M. (1966) Stress hormone metabolites and their fluctuations in the urine of rainbow trout (Salmo gairdneri) under the influence of various sublethal stressors. $\mathrm{Ph}$. D. dissertation, Univ. of Michigan.

Miles H. M. and Smith L. S. (1968). Ionic regulation in migrating juvenile salmon, Oncorhyncus kisutch. Comp. Biochem. Physiol. 26, 381-398.

Motais R., Romeu F. G. and Maetz J. (1965) Responses of flounder and serran on transfer to fresh water. C.r. hebd. Seanc, Acad. Sci. Paris 261, 801-804.

Potts W. T. W. and Fleming W. R. (1970) Hormone effects on ion and water regulation in Fundulus. J. exp. Biol. 53, 317-327.

Shanes A. M. (1960) Mechanism of change in permeability in living membranes. Nature 188, $1209-1210$.

Scharatt B. M., Jones I. C. and Bellamy D. (1964) Water and electrolyte composition of the body and renal function of the eel (Anguilla anguilla L.). Comp. Biochem. Physiol. 11, 9-18.

Smith R. I. (1970) Chloride regulation at low salinities by Neresis. J. exp. Biol. 53, 75-92.

Talbot C., Eddy F. B. and Johnston J. (1982) Osmoregulation in salmon and seatrout alevins. J. exp. Biol. $101,61-70$.

Thorson T. (1961) The partitioning of body water in Ostcichthycs: Phylognetic and ccological implications in aquatic vertebrates. Biol. Bull. Mar. Biol. Lab. Woods Hole 120, 238-254.

Weisbart M. (1967) Osmotic and ionic regulation in embryos, alevins and fry of five species of Pacific salmon. Ph.D. dissertation, Univ. of British Columbia, $94 \mathrm{pp}$.

Wood C. M. and Randall D. J. (1973a) The influence of swimming activity on $\mathrm{Na}^{+}$balance in the rainbow trout (Salmo gairdneri) J. Comp. Physiol. 82, 207-234.

Wood C. M. and Randall D. J. (1973b) The influence of swimming activity on water balance in the rainbow trout (Salmo gairdneri) J. Comp. Physiol. 82, 257-276. 